\title{
Association between Recurrence and Survival Rates According to the Location of Gastric Gastrointestinal Stromal Tumor
}

\author{
Yeong Ji Yu, Tae Ho Kim, Jung Hwan Oh, Woo Chul Chung \\ Department of Internal Medicine, College of Medicine, The Catholic University of Korea, Seoul, Korea
}

Background/Aims: This study aimed to evaluate the clinicopathological parameters of gastric gastrointestinal stromal tumors (GISTs) and to investigate the effect of tumor site on clinical outcomes.

Materials and Methods: Patients treated for a surgically confirmed gastric GIST were retrospectively evaluated between January 2001 and June 2016. The risk level was determined on the basis of the tumor size and number of mitoses. The risk level, recurrence rate, and survival rates were evaluated on the basis of the site of the gastric GISTs.

Results: The 97 patients identified included 42 men and 55 women. The median follow-up period was 36 months (range: 12 72 years). Of the 97 patients, $57(58.7 \%)$ and $40(41.3 \%)$ patients had proximally and distally located gastric GISTs, respectively. The high- and low-risk groups had higher proportions of proximally and distally located tumors, respectively $(P=0.033)$. The recurrence rates of proximal and distal GISTs were $8.8 \%$, and $2.5 \%$, respectively $(P=0.307)$. The overall survival rate was not associated with the site of the gastric GISTs $(P=0.549)$.

Conclusions: No relevant associations were found among recurrence, overall survival, and gastric GIST location. (Korean J Helicobacter Up Gastrointest Res 2019;19:169-173)

Key Words: Follow-up studies; Gastrointestinal stromal tumors; Retrospective studies; Stomach neoplasms; Survival rate

\section{INTRODUCTION}

Gastrointestinal stromal tumor (GIST) is a commonly occurring mesenchymal tumor of the gastrointestinal system and consist of interstitial cells of Cajal (ICC) or their stem cells that activate mutations of KIT or PDGFRA protooncogenes that are involved in oncogenicity of GISTs. ${ }^{1,2}$ GISTs occur mainly in adults and the median age is 55 to 60 years. ${ }^{3}$ About $80 \%$ of GISTs are expressed as mesenchymal tumors and account for less than 3\% of all gastrointestinal malignancies. ${ }^{4-7}$ The most common sites for GIST are stomach (70\%), small intestine (20 30\%), colon (5 10\%) and esophagus (5\%). ${ }^{8}$ Although the Swedish population-based study $^{3}$ and the pathological study of the Armed Forced Institute of Pathology ${ }^{9}$ have been reported, there is limited research on the relevance and outcomes of gastric GISTs risk according to the location of gastric GISTs. Previous stud-

Received: February 28, 2019 Revised: May 6, 2019 Accepted: May 8, 2019

Comesponding author: Jung Hwan Oh

Department of Internal Medicine, Eunpyeong St. Mary's Hospital, College of Medicine, The Catholic University of Korea, 1021 Tongil-ro, Eunpyeong-gu, Seoul 03312, Korea Tel: +82-2-2030-2535, Fax: +82-2-2030-4641, E-mail: ojh@catholic.ac.kr

The study was approved by the Institutional Review Board of the Catholic University of Korea (PC18RESI0042) ies have concentrated on the overall GIST of the whole. There were several clinicopathologic studies focused on gastric GISTs, but they were performed in small groups. ${ }^{10-12}$ The prognosis of patients with GISTs might be related to the anatomical site of the tumor. ${ }^{13}$ The incidence of gastric GISTs is known to be generally low in lower third of the stomach. ${ }^{10-12}$ However, these studies did not elucidate the reasons for the incidence rates based on the location of gastric GISTs. In order to determine whether the site of GISTs in the stomach affects a specific prognosis, further analysis of the clinical outcome according to the GIST location in the stomach is necessary. This study was designed to predict the clinical outcome based on the site of gastric GISTs retrospectively in multicenter and to identify clinicopathologic factors and survival rates of patients with primary gastric GISTs.

\section{MATERIALS AND METHODS}

\section{Patients}

This multicenter, retrospective study was performed at three Catholic University Hospitals in Korea. Between

Copyright $\odot 2019$ Korean College of Helicobacter and Upper Gastrointestinal Research

(a) The Korean Journal of Helicobacter and Upper Gastrointestinal Research is an Open-Access Journal. All articles are distributed under the terms of the Creative Commons Attribution Non-Commercial License (http:// creativecommons.org/licenses/by-nc/4.0) which permits unrestricted non-commercial use, distribution, and reproduction in any medium, provided the original work is properly cited. 
January 2001 and June 2016, the medical records of 97 GIST patients who were confirmed by surgery were reviewed. Medical records were reviewed for clinical parameters, including gender, age, tumor site, symptoms, surgery, chemotherapy, and pathological characteristics. The patients were subdivided into three groups. The location of gastric GISTs was divided anatomically into upper third, middle third and lower third portion. The upper third and middle third parts were then combined to form the proximal portion and the lower third to the distal portion.

\section{Pathology}

Immunohistochemical staining analysis was performed to identify immunophenotypes. Immunohistochemistry processing was performed via antibodies against CD117 (c-kit), S-100 protein and CD-34 and SMA-alpha. Almost all patients were confirmed as gastric GISTs by immunohistochemical staining for CD117 (c-kit), CD34, or both. The definition of pathological diagnosis in GISTs, which is confirmed by cell morphology and immunohistochemical staining. The risk was classified as very low, low, intermediate, and high according to National Institute of Health (NH) risk-group stratification system. ${ }^{1}$ Pathological parameters were evaluated, including mitotic count, tumor size, and cellularity. The tumor size was measured as the longest length. The mitotic counts were evaluated on 50 high power fields. Eophagogastroduodenoscopy and CT were done as a tool for preoperative examination.

\section{Prognosis analysis}

The survival period was evaluated from the day of surgery to death, related to the disease or not. In addition, this period was calculated based on whether it was related to the disease and whether it was censored for the latest follow-up observations. Follow-up studies were

Table 1. Comparison of the Distally and Proximally Located Gastric GastroIntestinal Stromal Tumors

\begin{tabular}{|c|c|c|c|c|}
\hline Variables & Total $(\mathrm{n}=97)$ & Distal $(n=40)$ & Proximal $(n=57)$ & $P$-value \\
\hline Age & $64.3 \pm 12.3$ & $62.0 \pm 13.3$ & $66.0 \pm 11.4$ & 0.114 \\
\hline Sex & & & & 0.241 \\
\hline Female & $55(56.7)$ & $26(65.0)$ & $29(50.9)$ & \\
\hline Male & $42(43.3)$ & $14(35.0)$ & $28(49.1)$ & \\
\hline Longest length of tumor $(\mathrm{cm})$ & $5.0 \pm 3.3$ & $4.1 \pm 3.0$ & $5.7 \pm 3.3$ & 0.019 \\
\hline NIH risk & & & & 0.033 \\
\hline Very low & 29 (29.9) & $16(40.0)$ & $13(22.8)$ & \\
\hline Low & 29 (29.9) & $14(35.0)$ & $15(26.3)$ & \\
\hline Intermediate & 19 (19.6) & $7(17.5)$ & $12(21.1)$ & \\
\hline High & $20(20.6)$ & $3(7.5)$ & $17(29.8)$ & \\
\hline Symptoms & & & & 0.395 \\
\hline Yes & $45(46.4)$ & $16(40.0)$ & $29(50.9)$ & \\
\hline No & $52(53.6)$ & $24(60.0)$ & $28(49.1)$ & \\
\hline Recurrence & & & & 0.307 \\
\hline Yes & $6(6.2)$ & $1(2.5)$ & $5(8.8)$ & \\
\hline No & $90(92.8)$ & $39(97.5)$ & $51(89.5)$ & \\
\hline Unknown & $1(1.0)$ & $0(0.0)$ & $1(1.8)$ & \\
\hline Survival & & & & 0.549 \\
\hline Yes & $58(60.4)$ & $21(53.8)$ & 37 (64.9) & \\
\hline No & $4(4.2)$ & $2(5.1)$ & $2(3.5)$ & \\
\hline Unknown & $35(35.4)$ & $17(41.1)$ & $18(31.6)$ & \\
\hline
\end{tabular}

Values are presented as mean \pm standard deviation or $\mathrm{n}(\%)$.

$\mathrm{NIH}$, National Institutes of Health. 

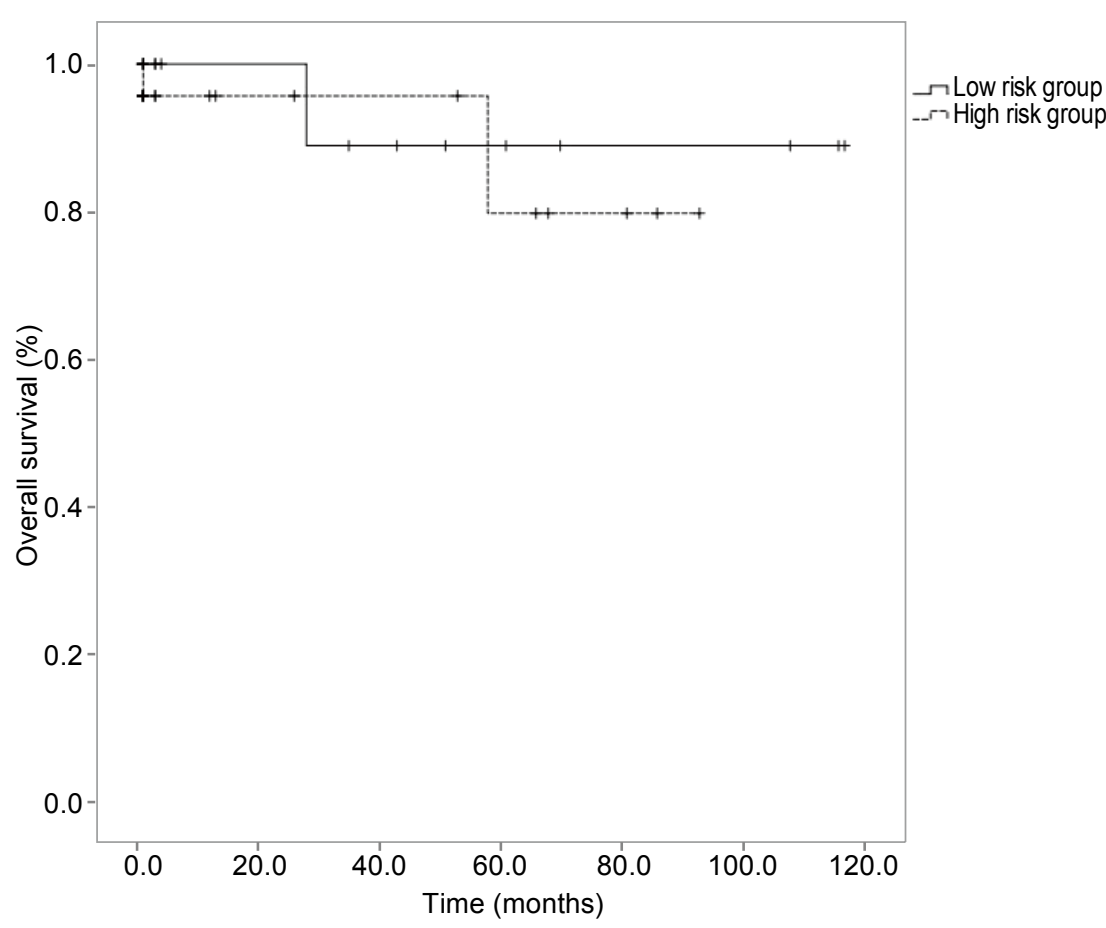

Fig. 1. Kaplan-Meier curves of survival probability rates according to two risk groups of gastric gastrointestina stromal tumors. No signi ficant differences are observed in overall survival between the two groups of patients $(P=0.618)$.

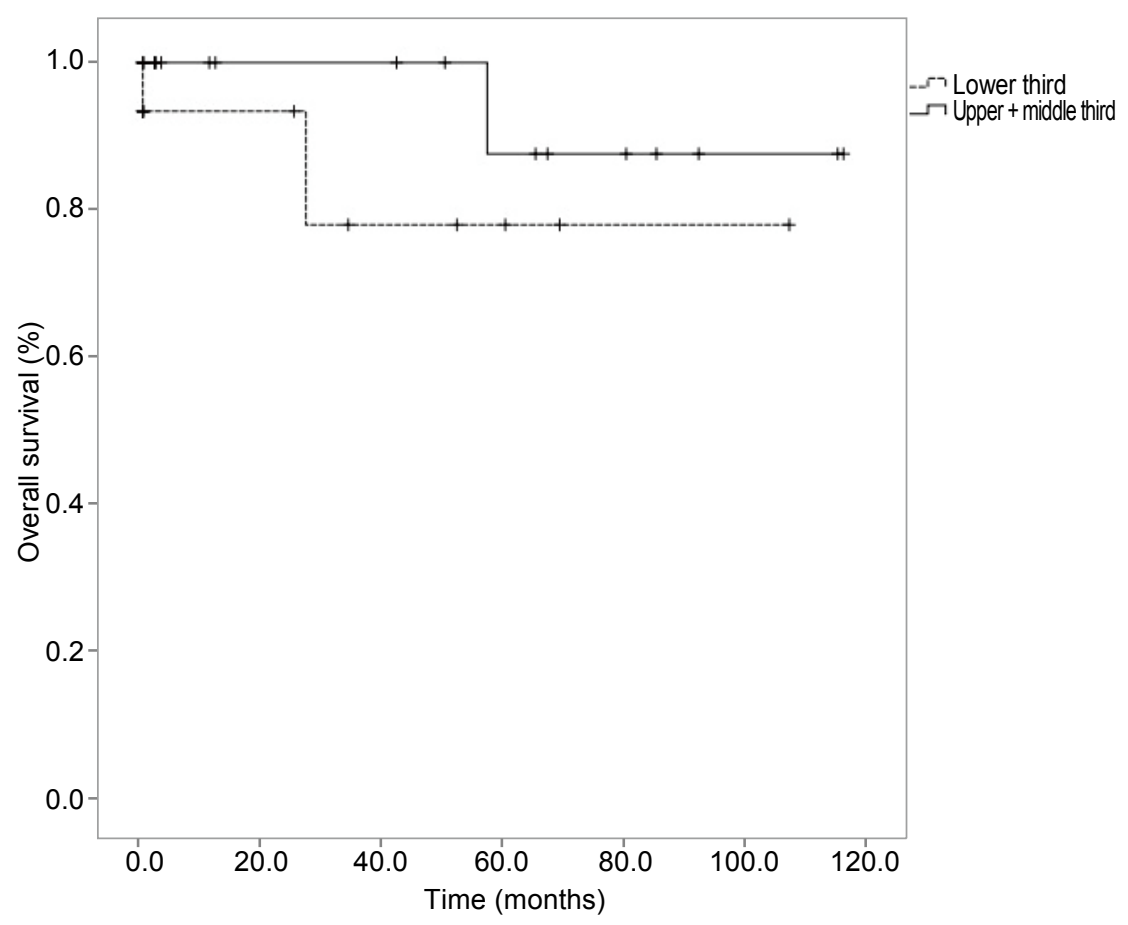

Fig. 2. Kaplan-Meier curves of survival probability rates according to the two sites of gastric gastrointestina stromal tumors. No statistically significant difference is observed between the proximally (upper third+middle third part of the stomach) and distally located gastric gastrointestinal stromal tumors (lower third part of the stomach; $P=0.237$ ).

performed as follows: chest X-ray, esophagogastroduodenoscopy, abdominal CT scans, chest CT scans, and/or PET scans. Recurrence was assessed according to the medical records. Tumor sites of the stomach and risk group were used for survival analysis.

\section{Statistical analysis}

Categorical data were presented as numbers (\%). They were compared using the chi-square test. Continuous variables were represented as the mean \pm standard devia- 
tion, and were compared between groups using the Student's t-test. Survival curves were constructed using a Kaplan-Meier survival analysis with comparisons between the curves based on a log-rank $\chi^{2}$ statistic. A $P$-value of less than 0.05 was considered to be statistically significant. Statistical analysis was performed using SPSS $^{\circledR}$ Statistics 21.0 (IBM, Armonk, NY, USA).

\section{RESULTS}

\section{Clinical characteristics}

Ninety seven patients were diagnosed with primary gastric GISTs. There were 42 males (43.3\%) and 55 females $(56.7 \%$ ), with a mean age of $64.3 \pm 12.3$ (SD) years. While $45(46,4 \%)$ patients had symptoms such as abdominal pain, dyspepsia, fatigue, palpable mass, gastrointestinal bleeding and anemia, the rest of them were asymptomatic, incidentally found during an annual screening examination or evaluation of other disease.

\section{Histopathologic features}

Average longest length of tumor is $5.0 \pm 3.3(\mathrm{SD}) \mathrm{cm}$. Immunohistochemical staining demonstrated that 82 tumors (86.3\%) were positive for CD117 and 83 (85.6\%) were positive for CD34. The patients was classified by risk group according to the $\mathrm{NH}$ criteria. Based on the $\mathrm{NH}$ criteria, 29.9\% of patients were distributed in the very low risk group, $29.9 \%$ were in the low risk group, $19.6 \%$ were intermediate risk, and $20.6 \%$ were high risk. Four risks were divided into low risk and high risk. The low-risk group had low or very low risk patients and the high-risk group included patients with an intermediate or high risk.

\section{Tumor site and outcome}

Of the 97 tumors, 57 (58.3\%), and 40 (41.7\%) were located in the proximal and the distal part of the stomach, respectively. The median follow-up period was 36 months (range: 12 72). Table 1 compared the characteristics in distal and proximal portions of the gastric GISTs, which indicated that proximally located tumors had much more cases of high risk group than distal tumors $(P=0.033)$. The average length of tumor was statistically significantly longer in the proximal part of the tumor than in the distal part $(P=0.019)$. The recurrence rate of proximally located GISTs was $8.8 \%$, and for distally located GISTs, it was $2.5 \%(P=0.307)$. In addition, the overall survival rate did not correlate with the site of the gastric GISTs $(P>0.999)$.

\section{Prognostic evaluation}

The patients were classified into two groups based on risk of gastric GISTs. There was no significant difference in overall survival between the two groups of patients in Kaplan-Meier curve ( $P=0.618$ ) (Fig. 1). The survival curve was also analyzed according to the location of GISTs in the stomach. There was no statistically significant difference between the proximal part (upper third+middle third part of the stomach) and the distal part (lower third part of the stomach) of the gastric GISTs ( $P=0.237$ ) (Fig. 2).

\section{DISCUSSION}

In this study, we assessed the recurrence and survival rate according to the location of gastric GISTs, however, we did not demonstrate the usefulness of the location of gastric GISTs in assessing the risk and prognosis. We found the large size of the GIST tumors in the proximal portion of the stomach than in the distal stomach.

As prognostic factors of GISTs, the following facts were revealed: age, sex, tumor site, tumor size, incomplete resection, mitotic count, KIT mutations, mucosal involvement, operative variables, proliferative index, and epithelioid subtype. Among these, mitotic activity, tumor size, risk stratification and complete surgical resection were essential prognostic parameters of primary GISTs, which was confirmed in several studies. ${ }^{14,15}$ Based on the $\mathrm{NIH}$ risk criteria for tumor sizes and mitotic counts, the proximal portion of the stomach may be at higher risk because the size of the tumor is larger in the proximal portion of the stomach. The number of mitotic counts is known to be the most important parameter to determine the malignant tumor in the prognosis of GISTs and high 
mitotic activity is known to be a sign of active growth and tumor malignancy. Therefore, the higher the mitotic count, the more likely it is to develop malignancy tumor, which is likely to affect the prognosis of GIST patients. If there would be evidence of other malignant features despite of low mitotic count, a malignant GISTs should still be diagnosed. Tumor size is only marginally correlated with malignant tumors and is less important than histopathological factors. ${ }^{16}$ In a study analyzed 1,756 cases of gastric GISTs, it was found that the location of gastric GISTs in the fundus or gastroesophageal junctions was unfavorable factors, whereas it was favorable factors in the antrum. ${ }^{17}$ One possibility is that the changes in the proliferative properties of ICC cells and stromal smooth muscle stem cells may differ depending on the anatomical location of the stomach.

There are several limitations to our study. We could not evaluate the risk by subdividing the mitotic count and tumor size according to the site of gastric GISTs. The patients who failed to follow up after surgery was relatively high, and mutation analysis and pathologic analysis of GISTs were not performed in this study.

In conclusion, there is no relevance between the recurrence rate, the overall survival rate, and the site of the gastric GISTs in our study. The proximal GISTs may be an unfavorable factor, it seems that the proximal portion of gastric GISTs is larger than the distal portion.

\section{CONFLICT OF INTEREST}

No potential conflict of interest relevant to this article was reported.

\section{ORCID}

$\begin{array}{ll}\text { Yeong Ji Yu } & \text { (D) https://orcid.org/0000-0001-7010-1462 } \\ \text { Tae Ho Kim } & \text { (1) https://orcid.org/0000-0003-2015-5176 } \\ \text { Jung Hwan Oh } & \text { (1) https://orcid.org/0000-0002-9274-882X } \\ \text { Woo Chul Chung } & \text { (1) https://orcid.org/0000-0003-1044-0440 }\end{array}$

\section{REFERENCES}

1. Fletcher CD, Berman JJ, Corless C, et al. Diagnosis of gastro- intestinal stromal tumors: a consensus approach. Hum Pathol 2002;33:459-465.

2. Heinrich MC, Corless CL, Demetri GD, et al. Kinase mutations and imatinib response in patients with metastatic gastrointestinal stromal tumor. J Clin Oncol 2003;21:4342-4349.

3. Nilsson B, Bümming P, Meis-Kindblom JM, et al. Gastrointestinal stromal tumors: the incidence, prevalence, clinical course, and prognostication in the preimatinib mesylate era--a population-based study in western Sweden. Cancer 2005;103: 821-829.

4. Burkill GJ, Badran M, Al-Muderis O, et al. Malignant gastrointestinal stromal tumor: distribution, imaging features, and pattern of metastatic spread. Radiology 2003;226:527-532.

5. DeMatteo RP, Lewis JJ, Leung D, Mudan SS, Woodruff JM, Brennan MF. Two hundred gastrointestinal stromal tumors: recurrence patterns and prognostic factors for survival. Ann Surg 2000;231:51-58.

6. Duffaud F, Blay JY. Gastrointestinal stromal tumors: biology and treatment. Oncology 2003;65:187-197.

7. Lewis JJ, Brennan MF. Soft tissue sarcomas. Curr Probl Surg 1996;33:817-872.

8. Miettinen M, Lasota J. Gastrointestinal stromal tumors: review on morphology, molecular pathology, prognosis, and differential diagnosis. Arch Pathol Lab Med 2006;130:1466-1478.

9. Miettinen M, Makhlouf H, Sobin LH, Lasota J. Gastrointestinal stromal tumors of the jejunum and ileum: a clinicopathologic, immunohistochemical, and molecular genetic study of 906 cases before imatinib with long-term follow-up. Am J Surg Pathol 2006:30:477-489.

10. An JY, Choi MG, Noh JH, et al. Gastric GIST: a single institutional retrospective experience with surgical treatment for primary disease. Eur J Surg Oncol 2007;33:1030-1035.

11. Catena F, Di Battista M, Fusaroli P, et al. Laparoscopic treatment of gastric GIST: report of 21 cases and literature's review. J Gastrointest Surg 2008;12:561-568.

12. Mochizuki Y, Kodera Y, Ito S, et al. Treatment and risk factors for recurrence after curative resection of gastrointestinal stromal tumors of the stomach. World J Surg 2004;28:870-875.

13. Rabin I, Chikman B, Lavy R, et al. Gastrointestinal stromal tumors: a 19 year experience. Isr Med Assoc J 2009;11:98-102.

14. Hassan I, You YN, Shyyan R, et al. Surgically managed gastrointestinal stromal tumors: a comparative and prognostic analysis. Ann Surg Oncol 2008;15:52-59.

15. Rutkowski P, Nowecki ZI, Michej W, et al. Risk criteria and prognostic factors for predicting recurrences after resection of primary gastrointestinal stromal tumor. Ann Surg Oncol 2007; 14:2018-2027.

16. Hou YY, Lu SH, Zhou Y, et al. Predictive values of clinical and pathological parameters for malignancy of gastrointestinal stromal tumors. Histol Histopathol 2009;24:737-747.

17. Miettinen M, Sobin LH, Lasota J. Gastrointestinal stromal tumors of the stomach: a clinicopathologic, immunohistochemical, and molecular genetic study of 1765 cases with long-term follow-up. Am J Surg Pathol 2005;29:52-68. 